\title{
A functional vagotomy induced by unilateral forced right nostril breathing decreases intraocular pressure in open and closed angle glaucoma
}

Mount Pleasant Hospital Addiction Studies Foundation, PO Box 16336, Jerusalem, Israel

J Backon

Department of Ophthalmology, Hadassah University Hospital, Jerusalem, Israel

N Matamoros

M Ramirez

R M Sanchez

J Ferrer

A Brown

U Ticho

Correspondence to:

Dr J Backon.

Accepted for publication

11 May 1990

Joshua Backon, Nelson Matamoros, Manuel Ramirez, Rodrigo M Sanchez, Jaime Ferrer, Alberto Brown, Uriel Ticho

\begin{abstract}
There is evidence of the central regulation of intraocular pressure, and it has been suggested that vagal tone might be increased in glaucoma simplex. The nasal cycle, the simultaneous congestion-decongestion response in the nasal cavities, reflects the dynamic lateralisation of the autonomic nervous system. Since this lateralisation presents with sympathetic activity induced by left brain hemisphere stimulation and parasympathetic activity induced by right hemisphere stimulation, it was subsequently demonstrated that forced unilateral nostril breathing induces selective contralateral hemispheric stimulation as measured by relative increases in the electroencephalographic amplitude in the contralateral hemisphere as well as alternating lateralisation of plasma catecholamines. Using this functional vagotomy, we report that left hemispheric stimulation by 20 minutes of forced unilateral right nostril breathing led to a significant bilateral decrease of $4.6 \mathrm{mmHg}$ $(25 \%)$ in intraocular pressure in 46 patients with open and closed angle glaucoma. However, it significantly increased the IOP in three patients with neovascular, one with juvenile onset, and one with closed angle glaucoma.
\end{abstract}

Adrenergic influences on aqueous humour dynamics and intraocular pressure are well documented, ${ }^{1-3}$ and it has been suggested that vagal tone may be raised in glaucoma simplex. ${ }^{45}$

The nasal cycle, the simultaneous congestiondecongestion response in the nasal cavities, reflects the dynamic lateralisation of the autonomic nervous system. ${ }^{6}$ Since this lateralisation presents with sympathetic activity induced by left brain hemisphere stimulation and parasympathetic activity induced by right hemisphere stimulation, it was subsequently demonstrated that forced unilateral nostril breathing induces selective contralateral hemispheric stimulation as measured by relative increases in the electroencephalographic (EEG) amplitude in the contralateral hemisphere ${ }^{7}$ as well as alternating lateralisation of plasma catecholamines. ${ }^{8} \mathrm{We}$ have used forced unilateral nostril breathing as a functional vagotomy/sympathectomy in the investigation of autonomic parameters such as $T$ and $Q R S-T$ axis vectors in the electrocardiogram, stroke volume, parotid salivary flow rate, and parotid salivary immunoglobulins, glycaemic homoeostasis,' pupillary dilation, hippus and pupil cycle time, transcutaneously measured blood gases, blink rates, ${ }^{10}$ pulse wave velocity, and vagal tone as measured by spectral analysis of R-R intervals of the electrocardiogram. Using this functional vagotomy/sympathectomy in an experimental design, we recently reported the novel finding that right hemispheric activation via forced unilateral left nostril breathing increased the IOP by an average of $4.5 \%$, whereas left hemispheric stimulation via forced unilateral right nostril breathing led to a significant (25\%) decrease in IOP." We subsequently used a functional vagotomy via forced unilateral right nostril breathing to lower the IOP by an average of $4 \mathrm{mmHg}$ in 68 normal volunteers. ${ }^{12}$ We now report the results of our technique in glaucoma patients.

\section{Material and methods}

We investigated the effect of forced unilateral right nostril breathing on the IOP in 51 patients with diagnosed glaucoma ( 31 males, 20 females, aged 15-78). There were 33 subjects with open angle, 14 with closed angle including two with acute closed angle, three with neovascular, and one with juvenile glaucoma. After baseline testing of the IOP each subject underwent 20 minutes of forced unilateral right nostril breathing, with the contralateral nostril stuffed with cotton packing. To guard against observer bias the junior registrar who did the applanation tonometry was not told why the subject had his nostril stuffed. All patients were under standard pharmacological treatment, and some had even undergone surgical intervention.

Both parametric and non-parametric tests were used to determine statistical significance. All statistical results report 2-tailed values, except for the binomial test, where 1-tailed results are reported.

\section{Results}

As ascertained by applanation tonometry, we found a significant $(\mathrm{p}<0.0001)$ reduction of IOP with forced unilateral right nostril breathing even after pharmacotherapy and/or surgical intervention. Apart from the findings in the 'outliers' (the three with neovascular glaucoma, the one with juvenile, and the one with closed angle) the IOP was bilaterally lowered an average of $4.6 \mathrm{mmHg}$ from baseline. This decrease was $5.26 \mathrm{mmHg}$ in the right eye and $4.24 \mathrm{mmHg}$ in the left. Contraindications were not found and no side effects were reported. Higher baseline values of IOP resulted in a significantly greater effect of treatment. The baseline IOP was arbitrarily dichotomised as $20 \mathrm{mmHg}$ or lower and 
$21 \mathrm{mmHg}$ or higher. When the baseline IOP of the right eyes was greater than $21 \mathrm{mmHg}$, the median difference after treatment was $8 \mathrm{mmHg}$ ( $32 \%$ difference), whereas if the baseline was lower than $21 \mathrm{mmHg}$ the median difference was only $4 \mathrm{mmHg}$ ( $23 \%$ difference). When the baseline IOP of the left eyes was greater than $21 \mathrm{mmHg}$ the median difference after treatment was $6 \mathrm{mmHg}(27 \cdot 3 \%$ difference), whereas if lower than $21 \mathrm{mmHg}$ the median difference was only $3.5 \mathrm{mmHg}$ (19.5\% difference). The 'outliers' were included in these calculations. Overall, the average percentage decrease in IOP was $27 \%$ in the right eyes and $22 \cdot 5 \%$ in the left.

Omega-squared $\left(\omega^{2}\right)$ is one of the most frequently applied methods in estimating the proportion of the dependent variable accounted for by an independent variable, and it is used to confirm the strength of association between variables in a population. ${ }^{13}$ Here $\omega^{2}$ was $21.5 \%$ for the right eyes and $17 \cdot 2 \%$ for the left. The 'outliers' were included in these calculations.

Reliability in repeated applanation tonometry was investigated in 1972. Repeated Goldmann

Table 1 Effect of 20 min of forced unilateral right nostril breathing on the IOP (all groups combined)

\begin{tabular}{llcc}
\hline & & Right eye & Left eye \\
\hline Baseline & & & \\
& Mean & 20.15 & 18.71 \\
& Median & 19.00 & 18.00 \\
& SD & 6.13 & 4.03 \\
After 20 & & \\
minutes of FURNB & Mean & 16.31 & 15.64 \\
& Median & 14.00 & 15.00 \\
& SD & 7.04 & 6.43 \\
\hline
\end{tabular}

$\mathbf{n}=51$.

One-way ANOVA: both eyes $\mathrm{p}=<0.00048$

One-way ANOVA: right eye $p=<0.0040$

One-way ANOVA: left eye $p=<0.0049$

Signed rank test $p=<0.0000034$

Binomial test: at $50 \% \mathrm{p}=<0.000001$; at $33 \% \mathrm{p}=<0.000001$

Pitman randomisation test $\mathrm{p}=<0.00001$.

$\star$ Forced unilateral right nostril breathing.

Table 2 Effect of 20 minutes of forced unilateral right nostril breathing on the IOP in glaucoma subcategories.

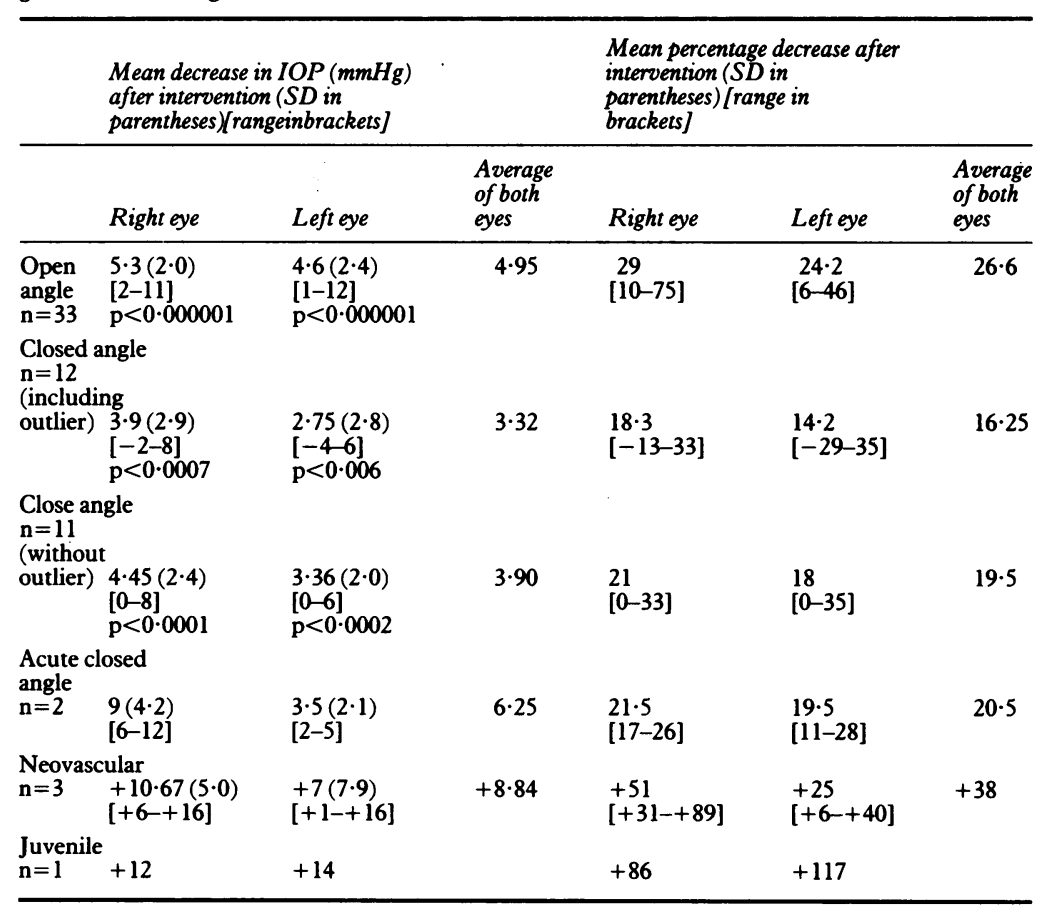

+ Signifies increase. applanation tonometry every minute for 5 minutes in normal subjects gave a direct pressure reduction of approximately $2.62 \mathrm{mmHg}$ in 5 minutes, whereas applanation tonometry carried out twice in a 5-minute period reduced the IOP only by $0.4 \mathrm{mmHg} .{ }^{14}$ Since the normal mean IOP is $15.4 \mathrm{mmHg}$ (SD 2.5), this gives a reliability of at least $83 \%(2 \cdot 62 / 15 \cdot 4)$. The percentage change in our investigation was on average much higher than $17 \%$. In order to verify that the intervention of forced unilateral nostril breathing was in fact the factor inducing the change in IOP, we measured the IOP twice in a 15 -minute period in another group of 12 patients with open angle glaucoma. Interestingly, the mean change in IOP in both left and right eyes was $0.417 \mathrm{mmHg}$. The percentage change was $5 \cdot 1 \%$ in the right eyes and $2.5 \%$ in the left. Both measurements were highly correlated (Pearson $r=0.99$ ), and neither parametric nor non-parametric testing revealed any statistically significant differences between measurements.

However, the IOP was significantly increased after forced unilateral right nostril breathing in the three subjects with neovascular glaucoma, the one subject with juvenile glaucoma, and one subject with closed angle glaucoma. The IOP decreased in all other patients, including the subject with acute closed angle glaucoma.

Statistical analysis by both parametric and non-parametric tests are indicated in Table 1 (all groups combined). Breakdown by category appears in Table 2 along with an appropriate statistical analysis.

\section{Discussion}

This study shows the fascinating autonomic regulation of intraocular pressure by forced unilateral nostril breathing. Such regulation has been demonstrated in an experimental study, ${ }^{1}$ in eyes with normal pressure, ${ }^{12}$ and now in patients with glaucoma. The decrease in values of IOP $(4.6 \mathrm{mmHg})$ by forced unilateral right nostril breathing in the glaucoma patients was similar to that found in the eyes of normal pressure. Pharmacological intervention (pilocarpine, acetazolamide) in glaucoma patients, however, is known to reduce the IOP by 1 torr $\left(133 \mathrm{~N} \mathrm{~m}^{-2}\right)$ over that lowered by pharmacological intervention in eyes of normal pressure. We can offer no explanation why the forced breathing technique lowers the IOP to the same level in both normal people and glaucoma patients. The effect of forced unilateral right nostril breathing is almost immediate, and it induces a reduction in IOP of up to $30-35 \%$, as compared with pharmacotherapy, which takes effect over a period of 20-30 minutes and induces a change in IOP in the range of $15-35 \% .{ }^{15-17}$ What remains to be investigated is the exclusive use of the technique without the use of pharmacotherapy.

Keeping in mind the role of innervation to the ciliary body and outflow channels, we have not yet explored whether the technique inhibits the production and inflow of aqueous, or whether it activates the outflow pathways.

It is unclear why the technique greatly increased the IOP in the patients with juvenile and neovascular glaucoma. It is possible that forced 
unilateral right nostril breathing may not be effective in lowering the IOP in cases of juvenile and neovascular glaucoma because of the presence of major impediments to aqueous outflow.

Although the technique has a short-term, approximately 2-3-hour effect and cannot be offered as a clinical intervention, it may shed light on factors affecting chronic nasal resistance such as the effect of lateral recumbency, ${ }^{18-28}$ the presence of septal deviation and of nasal polyps. One of us (NM) has found some preliminary evidence of an increased incidence of nasal polyps in the right turbinate of glaucoma patients. The skin-pressure vegetative reflex, ${ }^{18}$ which affects nasal resistance, has been found to regulate parameters mediated by the autonomic nervous system. These factors need to be further studied in glaucoma patients.

1 Greaves DP, Perkins ES. Influence of the sympathetic nervous system on the intraocular pressure and vascular circulation of the eye. Brf Ophthalmol 1952; 36: 258-64.

2 Langham ME, Rosenthal AR. Role of the cervical sympathetic nerve in regulating intraocular pressure and circulation. $A m$ f Physiol 1966; 210: 786-94.

3 Belmonte C, Bartels SP, Liu JHK, Neufeld AH. Effects of stimulation of the ocular sympathetic nerves on IOP and aqueous humor flow. Invest Ophthalmol Vis Sci 1987; 28 1649-54.

4 Innemmee HC, van Zwieten PA. The central nervous influence of drugs on intraocular pressure. Rev Pure App Pharmacol Sci 1980; 1: 107-65.

5 Waitzman MB. Hypothalamus and ocular pressure. Suro Ophthalmol 1971; 16: 1-23.

6 Werntz DA, Bickford RG, Bloom FE, Shannahoff-Khalsa DS. Alternating cerebral hemispheric activity and the lateralization of autonomic nervous function. Hum Neurobiol lateralization of

7 Werntz DA, Bickford RG, Shannahoff-Khalsa DS. Selective hemispheric stimulation by unilateral forced nostril breathing. Hum Neurobiol 1987; 6: 165-71.

8 Kennedy B, Ziegler MG, Shannahoff-Khalsa D. Alternating lateralization of plasma catecholamines and nasal patency in humans. Life Sci 1986; 38: 1203-14.

9 Backon J, Kullok S. Changes in blood glucose levels induced by differential forced unilateral nostril breathing, a tech nique which affects both brain hemisphericity and autonomic activity. Med Sci Res 1988; 16: 1197-9.

10 Backon J, Kullok S. Effect of forced unilateral nostril breathing on blink rates: relevance to hemispheric lateralization of ing on blink rates: relevance to hemisph

11 Backon J, Matamoros N, Ticho U. Changes in intraocula pressure induced by differential forced unilateral nostril breathing; a technique that affects both brain hemisphericity and autonomic activity: a pilot study. Graefes Arch Clin Exp and autonomic activity: a pilot

12 Matamoros N, Backon J, Ticho U. The effect of differential brain hemisphericity, induced by forced unilateral nostril breathing, on regulation of intraocular pressure. In: de Oliveira LNF, ed. Ophthalmology today. Excerpta Medica International Congress Series 1988; 803: 679-80.

13 Torabi MR. How to estimate practical significance in health education research. $\mathcal{F}$ Sch Health 1986; 56: 232-4.

14 Wilke K. Effects of repeated tonometry: genuine and sham measurements. Acta Ophthalmol (KGh) 1972; 50: 574-82.

15 Hepler RS, Frankl IM. Marihuana smoking and intraocula pressure. F AMA 1971; 217: 1392 .

16 Makabe R. Ophthalmologische Untersuchungen mit dichlorphenyl-aminoimidazolin. Dtsch Med Wochenschr 1966; 91 1686-8.

17 Zimmerman TJ, Kaufman HE. Timolol, a beta-adrenergic blocking agent for the treatment of glaucoma. Arch Ophthalmol 1977; 95: 601-4.

18 Takagi K, Kobayasi S. Skin pressure-vegetative reflex. Acta Medica Biol 1955; 4: 3-57.

19 Cole P, Haight JSJ. Posture and the nasal cycle. Ann Otol Rhinol Laryngol 1986; 95: 233-7.
R

20 Davies AM, Eccles R. Reciprocal changes in nasal resistance to airflow caused by pressure applied to the axilla. Acta Otolaryngol (Stockh) 1985; 99: 154-9.

21 Haight JSJ, Cole P. Topographical anatomy of pressure points that alter nasal resistance. $\mathcal{F}$ Otolaryngol 1986; suppl 16 : $14-20$.

22 Kosaka M, Takagi K, Koyama M. Reflex inhibition of cold shivering by pressure in the skin and the histological investigation of its afferent spinal pathway. Experientia 1967; 23: 453-5.

23 Kumazawa T. 'Deactivation' of the rabbit's brain by pressure application to the skin. Electroencephalogr Clin Neurophysiol 1963; 15: 660-71.

24 Nakayama T. Behaviour of cervical muscles in labyrinthine and skin-pressure reflexes. $\mathcal{F}$ p $\mathcal{F}$ Physiol 1957; 7: 99-112.

25 Ogawa T, Asayama M, Ito M, Yoshida K. Significance of skin pressure in body heat balance. 7 pn $f$ Physiol 1979; 29: pressure

26 Rao S, Potdar A. Nasal airflow with body in various positions. f Appl Physiol 1970; 28: 162-5.

27 Singh V. Thoracic pressure and nasal patency. $f$ Appl Physiol 1987; 62: $91-4$

28 Takagi K. Über den Einfluss des mechanischen Hautdruckes auf die vegetativen Funktionen. Acta Neurovegetativa 1957; 16: 439-47. 